\title{
Analysis of Dental Hygienist Job Recognition Using Text Mining
}

\author{
Bo-Ra Kim ${ }^{1}$, Eunsuk Ahn², Soo-Jeong Hwang ${ }^{3}$, Soon-Jeong Jeong ${ }^{4}$, Sun-Mi Kim ${ }^{5}$, and \\ Ji-Hyoung $\operatorname{Han}^{6, \dagger}$ \\ 'Department of Dental Hygiene, College of Medical and Health Sciences, Cheongju University, Cheongju 28503, \\ ${ }^{2}$ Department of Dental Hygiene, Daejeon Institute of Science and Technology, Daejeon 35408, ${ }^{3}$ Department \\ of Dental Hygiene, College of Medical Science, Konyang University, Daejeon 35365, ${ }^{4}$ Department of Dental \\ Hygiene \& Institute of Basic Science for Well-Aging, Youngsan University, Yangsan 50510, ${ }^{5}$ Department of \\ Dental Hygiene, Wonkwang Health Science University, Iksan 54538, ${ }^{6}$ Department of Dental Hygiene, Suwon \\ Science College, Hwaseong 18516, Korea
}

\begin{abstract}
Background: The aim of this study was to analyze the public demand for information about the job of dental hygienists by mining text data collected from the online $Q$ \& A section on an Internet portal site.

Methods: Text data were collected from inquiries that were posted on the Naver Q \& A section from January 2003 to July 2020 using "dental hygienist job recognition," "role recognition," "medical assistance," and "scaling" as search keywords. Text mining techniques were used to identify significant Korean words and their frequency of occurrence. In addition, the association between words was analyzed.

Results: A total of 10,753 Korean words related to the job of dental hygienists were extracted from the text data. "Chi-lyo (treatment)," "chigwa (dental clinic)," "ske-illing (scaling)," "itmom (gum)," and "chia (tooth)" were the five most frequently used words. The words were classified into the following areas of job of the dental hygienist: periodontal disease treatment and prevention, medical assistance, patient care and consultation, and others. Among these areas, the number of words related to medical assistance was the largest, with sixty-six association rules found between the words, and "chi-lyo," "chigwa," and "ske-illing" as core words.

Conclusion: The public demand for information about the job of dental hygienists was mainly related to "chi-lyo," "chigwa," and "ske-illing" as core words, demonstrating that scaling is recognized by the public as the job of a dental hygienist. However, the high demand for information related to treatment and medical assistance in the context of dental hygienists indicates that the job of dental hygienists is recognized by the public as being more focused on medical assistance than preventive dental care that are provided with job autonomy.
\end{abstract}

Key Words: Awareness, Dental hygienist, Job description, Recognition, Text mining

\section{Introduction}

The Korean dental hygienist group has come to establish an academic basis for the reestablishment of the legal job of a dental hygienist in order to strengthen the institutional protection of the scope of the dental hygienists' actual job in Korean clinical settings ${ }^{1)}$. One of the external factors driving this movement is the public awareness of the jobs performed by dental hygienists. The job is a main factor recognizing dental hygienists to the public $^{2,3)}$. It has been reported that medical assistant of dentists' work $^{4-7)}$, scaling, and other preventive procedures ${ }^{3,7)}$ were the most common work practices that the general population received from dental hygienists. As the public 
awareness of the job of dental hygienists is related to dental treatment procedures which are not clearly defined in law, the public should have a clear awareness of the professional role of dental hygienists so that dental hygienists can perform legal job and ensure the public's oral health under the protection in law. For this, it is necessary to understand the current level of awareness and demand for information about dental hygienists by the public. Given that previous studies on this topic were conducted through interviews or panel surveys, and had disadvantages in terms of access to study participants and limited sample size, it is necessary to investigate information based on a large general population.

Social big data has been generated as a result of the rapid increase in the use of mobile Internet and social media in everyday life ${ }^{8)}$, and represents a source of public opinion ${ }^{9)}$. The messages in social big data such as social media and text messages contribute to the development of public policy by measuring public understanding and the sentiment of social issues based on the application of text mining, visualization, and statistical analysis ${ }^{10)}$.

Previous dental hygiene studies using social big data and text mining techniques have mainly reported the results of analysis of general information related to dental hygienists. A previous study analyzed the inquiries retrieved from the Q \& A section on an Internet portal site using the Korean names for dental hygienists. This study confirmed that the words used in most inquiries were related to entrance examination, employment, and the job of dental hygienists, depending on age groups ${ }^{11)}$. Another study analyzed inquiries posted by dental hygienists and the general public through various Internet sites, and reported that words relating to the job or career choice were used by the general public, and words relating to dental practice and turnover were used by dental hygienists $^{12)}$. This demand for information focused on the job and career of dental hygienists indicates a continued public interest in this job. However, at the same time, it also suggests that detailed information about the job is not widely-known across society. Thus, to facilitate accurate understanding of the jobs of dental hygienists, it is important to understand what information the general public requires, and to what extent. Therefore, we sought to analyze social big data related to the job of dental hygienists retrieved from online Q \& A sections on an Internet portal site using text mining techniques. This study confirmed the public's awareness and demand for information about the jobs of dental hygienists.

\section{Materials and Methods}

Text mining is a method of classifying and refining text data, and is used to discover meaningful information hidden in big data by analyzing the frequency of occurrence of keywords and summarizing the results ${ }^{9}$. As the first step after collecting the data of interest online, the text data are transformed to a form that the computer can understand using natural language processing. Next, stop words are removed, leaving only nouns that can be extracted as words. In this study, the frequency of occurrence of keywords, visualization of the frequency, and the association between the words were analyzed using this process.

\section{Data collection}

Data were collected from inquiries that were posted on the Q \& A section of the Naver which is a portal site, called Naver "Jishik-iN" from January 2003 to July 2020 using a web crawling technique. A data search was performed with "dental hygienist role recognition," "dental hygienist job recognition," "dental hygienist medical assistant," and "dental hygienist scaling" as keywords. The keywords were selected to search the contents and titles of inquiries that directly related to the jobs of dental hygienists. The words were also used in consideration of the fact that the most common hygienist services received by the public were medical assistance and scaling ${ }^{4,5)}$. Only the titles and contents of all retrieved inquiries (2,038 in total) were collected, and responses to the inquiries were excluded.

\section{Text preprocessing and analysis}

Preprocessing of the collected text data was performed using text mining techniques to extract and refine significant words. The text preprocessing workflow and examples are listed in Table 1. 


\section{1) Text natural language processing and primary refinement}

Natural language processing was performed to convert the collected text data into a form that can be understood by a computer (Table 1). This study analyzed Korean texts only, and as such, all English characters were excluded.

\section{2) Text morphological analysis and extraction}

Words related to the search keywords were extracted from the preprocessed text data using a dictionary function that extracted Korean words in the analysis program. Additional terminology, such as extracted words and 35 proper nouns (e.g., dental hygienists, medical aid), was added to the word extraction dictionary of the analysis program (Table 1). Thereafter, noun stem extraction (stemming) was performed through text morphological analysis, and 445,476 nouns were obtained as a result. Among these nouns, words with less than two letters, articles, prepositions, conjunctions, and words that were not directly related to the purpose of this study were classified as stop words and were removed (Table 1). A selection of 265,872 words was finally derived and used for text mining analysis.

\section{Analytic tools and text analysis sequence}

All data were collected and analyzed using $\mathrm{R}$ program (version 3.6.3, R Foundation for Statistical Computing, Vienna, Austria) ${ }^{13)}$. First, web crawling was performed to collect text data retrieved from the Naver "Jishik-iN" section, using the packages "rvest" and "dplyr" in $\mathrm{R}$ program. The collected text data were processed in their natural language using the package "KoNLP". Using the dictionary "Sejong" and "Woorimalsam", 35 nouns were added to the list of words to be extracted, and word extraction was performed based on the dictionary "NIA". After extracting the keywords related to the job of dental hygienists, a term-frequency matrix (TFM) was derived from the frequency of occurrence of each word, and a "word cloud" was composed to represent the frequency of the words as the size of character. Finally, the association rule mining technique was used to find associations between occurrence of two words, and the results were visualized.

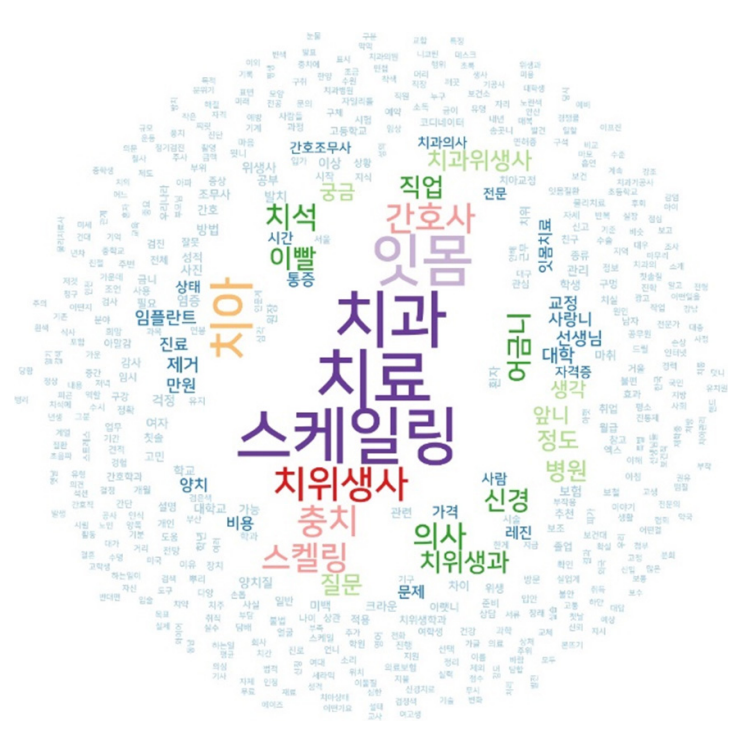

Fig. 1. Word cloud of the top 500 words.

Table 1. Workflow of Text Preprocessing and Examples

\begin{tabular}{|c|c|}
\hline Work & Examples \\
\hline Removal of special and numeral characters & 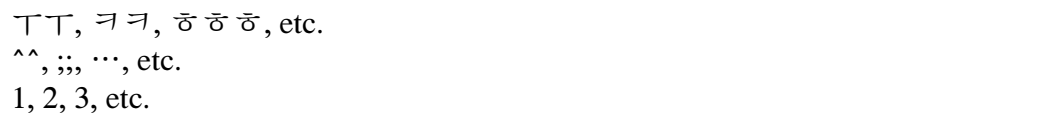 \\
\hline Addition of terminology and proper nouns $(n=35)$ & $\begin{array}{l}\text { Chigwawisaengsa , skelling, ganhojomusa, ske-illing, jomusa, eobmu, jigmu, } \\
\text { eobmubeom-wi, jigmubeom-wi, yeoghal, jinlyohyeobjo, jinlyobojo, } \\
\text { haegsim-yeoglyang, uilyoinhwa, uilyohaeng-wi, gam-yeom, gam-yeom-yebang, } \\
\text { insig, imiji, inji, uilyobunjaeng, suhaeng-eobmu, chigwagigongsa, chigigongsa, } \\
\text { chiwisaengsa, chiseog, chiwisaenghag-gwa, chiwisaeng-gwa, amalgam, } \\
\text { salangni, chigwa, gigongsa, bojo, seogsyeon, wisaengsa }\end{array}$ \\
\hline Removal of stopwords & $\begin{array}{l}\text { Dabbyeon, annyeong, oenjjog, naegong, butag, eumsig, oneul, etc. } \\
\text { Mueot, haeseo, guyo, hago, seyo, etc. } \\
\text { Geugeot, igeos, jeohui, etc. }\end{array}$ \\
\hline
\end{tabular}




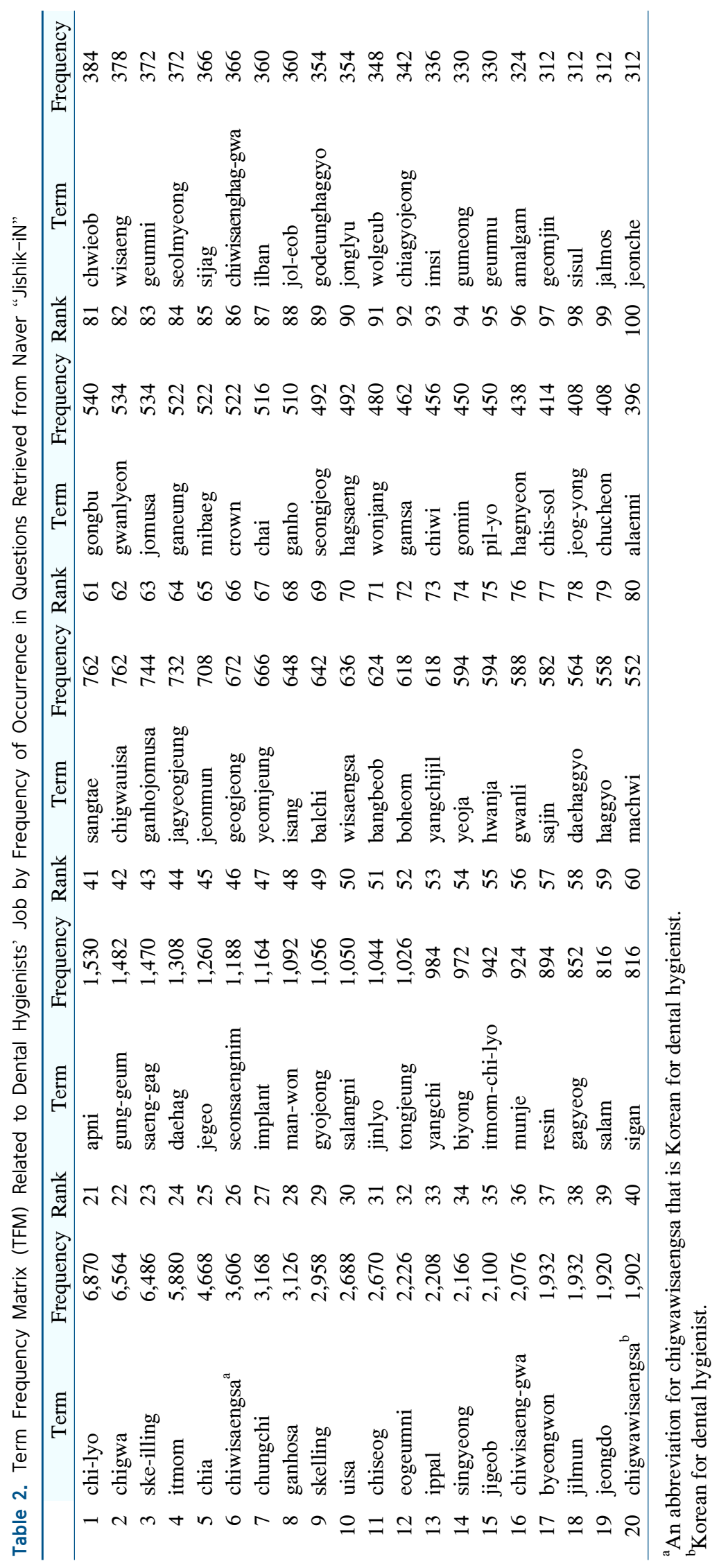




\section{Results}

\section{Results of word extraction and word cloud}

A total of 265,872 Korean words were collected from 2,038 inquiries retrieved from Naver "Jishik-iN." After composing a TFM by frequency occurrence of each word, a total of 10,753 different Korean words were identified. Fig. 1 shows a word cloud representing the 500 most frequently used words related to the job of dental hygienists. It can be seen that "chi-lyo" (treatment), "chigwa" (dental clinic), "ske-illing" (scaling), "itmom" (gum), "chia" (tooth), and "chiwisaengsa" (an abbreviation for chigwawisaengsa, which is Korean for dental hygienist) were the most frequently used words (Fig. 1).

\section{Frequently used words and their classification based on the job area of dental hygienists}

Table 2 lists the 100 most frequently used Korean words and their frequencies. By dividing the word by the job area of dental hygienists, "ske-illing" (scaling, 3rd), "itmom" (gum, 4th), "chiseog" (dental calculus, 11th), "itmom-chi-lyo" (treatment of the gingival or periodontal disease, 35th), and "yeomjeung" (inflammation, 47th) were the most frequently used words related to the treatment and prevention of periodontal disease.

The most frequently used words related to the medical assistant areas were "chungchi" (dental caries, 7th), "singyeong" (nerve, 14th), "implant" (27th), "gyojeong" (orthodontics, 29th), "jinlyo" (dental treatment or appointment, 31st), "resin" (37th), "balchi" (extraction, 49th), "machwi" (anesthesia, 60th), "crown" (66th), "geumni" (gold crown, 83rd), "imsi” (temporary, 93rd), and "amalgam" (96th). The number of words related to this work area was greater than that in other areas.

The most frequently used words related to patient care, cost consultation, and medical insurance claims were "biyong" (cost, 34th), "gagyeog" (price, 38th), "boheom" (insurance, 52nd), and "seolmyeong" (explanation, 84th).

In addition, "jeonmun" (specialty, 45th), "gwanli" (management, 56th), "mibaeg" (whitening, 65th), "gemjin" (examination, 97th), and "sisul" (procedure, 98th) were found as the job of dental hygienists-related words.

Regarding occupational titles, "chiwisaengsa" (Korean abbreviation for chigwawisaengsa, dental hygienist, 6th),

Table 3. Association Rules between Word Pairs in the Top 10 and Word Pairs of the Korean Words for Dental Hygienist (Chiwhisaengsa and Chigwawisaengsa)

\begin{tabular}{|c|c|c|c|c|c|}
\hline \multirow{2}{*}{ Rank $^{\mathrm{a}}$} & \multicolumn{2}{|c|}{ Association rules } & \multirow{2}{*}{ Support $^{\mathrm{b}}$} & \multirow{2}{*}{ Confidence $^{c}$} & \multirow{2}{*}{ Lift $^{\mathrm{d}}$} \\
\hline & Antecedent & Consequent & & & \\
\hline 1 & Seonsaengnim (sir) & Uisa (doctor) & 0.01 & 0.68 & 14.87 \\
\hline 2 & Jegeo (removal) & Chiseog (dental calculus) & 0.02 & 0.72 & 12.91 \\
\hline 3 & Singyeong (nerve) & Chi-lyo (treatment) & 0.03 & 0.86 & 8.35 \\
\hline 4 & Yeomjeung (inflammation) & Itmom (gum) & 0.01 & 0.81 & 7.13 \\
\hline 5 & Resin & Chi-lyo (treatment) & 0.01 & 0.64 & 6.23 \\
\hline 6 & Biyong (cost) & Chi-lyo (treatment) & 0.01 & 0.54 & 5.19 \\
\hline 7 & Uisa (doctor) & Chi-lyo (treatment) & 0.02 & 0.53 & 5.08 \\
\hline 8 & Chungchi (dental caries) & Chi-lyo (treatment) & 0.03 & 0.52 & 5.07 \\
\hline 9 & Seonsaengnim (sir) & Chigwa (dental clinic) & 0.01 & 0.68 & 4.90 \\
\hline 10 & Seonsaengnim (sir) & Chi-lyo (treatment) & 0.01 & 0.50 & 4.83 \\
\hline$\cdots$ & $\ldots$ & $\ldots$ & $\ldots$ & $\cdots$ & $\cdots$ \\
\hline 58 & Chiwisaengsa $^{\mathrm{e}}$ & Chigwa (dental clinic) & 0.02 & 0.35 & 2.54 \\
\hline$\ldots$ & $\cdots$ & $\ldots$ & $\cdots$ & $\cdots$ & $\cdots$ \\
\hline 66 & Chigwawisaengsa $^{\mathrm{e}}$ & Chigwa (dental clinic) & 0.01 & 0.31 & 2.26 \\
\hline
\end{tabular}

${ }^{\mathrm{a}}$ Ranks were determined according to the degree of lift value. The top 10 word-pairs show the confidence of association greater than 0.5 . ${ }^{\mathrm{b}}$ Support, probability of simultaneous occurrence of words A and B.

${ }^{\mathrm{C}}$ Confidence, probability of occurrence of word B when word A is appeared.

${ }^{\mathrm{d}} \mathrm{Lift}$, degree of association between words A and B. The higher lift value means that there is a higher probability of simultaneous occurrence of words A and B, comparing to probability of occurrence of the two words by chance ${ }^{14)}$.

${ }^{\mathrm{e}}$ Chiwisaengsa and chigwawisaengsa are Korean words for dental hygienist. 
"ganhosa" (nurse, 8th), "uisa" (doctor, 10th), "chigwawisaengsa" (dental hygienist, 20th), "seonsaengnim" (sir, 26th), "chigwauisa" (dentist, 42nd), "ganhojomusa" (nurse assistant, 43rd), and "wisaengsa" (hygienist, 50th) were identified as frequently used words.

Although "egseulei” (X-rays, 107th), "chisil” (dental floss, 117th), "bocheol" (prosthetics, 148th), "chiju" (periodontal, 163rd), "dambae "(cigarettes, 171st), "sodog" (disinfection, 180th), and "xylitol" (183rd) were not included in the list in Table 2, they were detected as noteworthy words related to the job of dental hygienists.

\section{Association rules between word pairs}

Table 3 shows the top 10 word pairs showing association rules according to the degree of association (lift value), in which the confidence of association is greater than 0.5 . The table also presents word pairs of the words "chiwisaengsa" and "chigwawisaengsa" (dental hygienist). Sixty-six rules were found as a result of the association analysis, and the words of each pair were positively associated (lift $>1$ ). This indicates that the occurrence of the two words is related. The degree of association between "seonsaengnim" and "uisa" (doctor) was the highest (lift=14.87). "Chi-lyo" had associations with multiple words (lift=4.83 8.35), so it was more likely to be exist with "singyeong," "resin," "biyong," "uisa," "chungchi," and "seonsaengnim." The words "chiwisaengsa" and "chigwawisaengsa" had association with "chigwa", and it was confirmed that these were likely to exist together.

Fig. 2 shows a networking map showing the information of 66 association rules. The arrow indicates the direction of association, and the circle size indicates the probability of simultaneous occurrence of the two words. The darker the circle color, the higher the association. The core words with the largest circle sizes were "chi-lyo," "chigwa," and "ske-illing." This indicates that the core words are often accompanied by other words. Moreover, high association

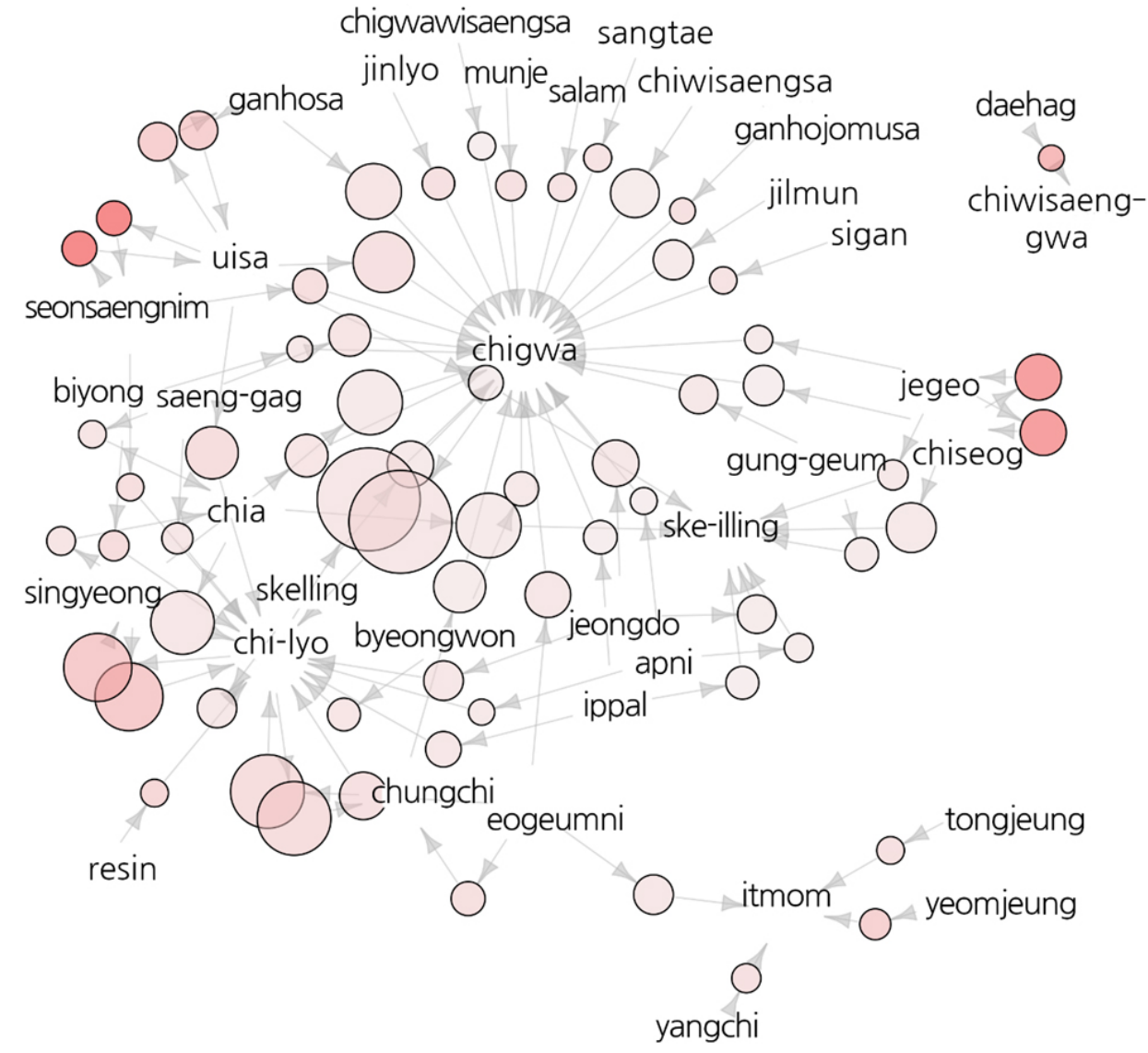

Fig. 2. Networking map reflecting 66 association rules between word pairs. 
was observed between "seonsaengnim" and "uisa," "chiseog" and "jegeo" (removal), and "daehag" (College) and "chiwisaeng-gwa" (Department of Dental Hygiene), based on darker circle colors. Two subgroups of association around the "itmom" and "chiwisaeng-gwa" were also observed in addition to the three core words-oriented associations.

\section{Discussion}

This study collected inquiries retrieved from Naver "Jishik-iN" with search words focused on the job and work of the dental hygienist. We extracted meaningful words from the text data using text mining techniques to confirm the public's awareness and demand for information about the job of dental hygienists. As a result, "chi-lyo" (treatment), "chigwa" (dental clinic), and "skeilling" (scaling) were found to be the most frequently used words. Moreover, it was confirmed that meaningful words related to treatment and prevention of periodontal disease, medical assistance, patient care and consultation, and others were used with high frequency in the inquiries when the extracted words were classified by the job areas of dental hygienists.

Dental calculus removal or scaling is highly valued by both dentists and dental hygienists ${ }^{15)}$. Moreover, several studies have reported that calculus removal or scaling is the most commonly performed job ${ }^{1,16}$, with $85.9 \%$ of medical workers ${ }^{17)}$ and $86 \%$ of prospective dentists ${ }^{18)}$ recognizing that the job is covered by dental hygienists. Corresponding to the recognition by experts, social recognition about scaling by the public was also found. Among the words extracted by text mining, "ske-illing" was the 3rd most frequently used word, and "skelling," which also means scaling in Korean, was the 9th most frequently used word. This result is encouraging given the active role of dental hygienists in the clinical setting against the background of institutional changes. The job performed and name tags are the most important factors in distinguishing dental hygienists from other occupational types $^{3,5,19)}$. In Korea, removal of dental calculus has been provided as an expansion of coverage since July 2013, and the use of name tags has been mandated with the implementation of medical service acts regarding the contents of name tags for medical personnel ${ }^{20)}$. The expansion of coverage to include scaling resulted in an increase in preventive dental care utilization ${ }^{21,22)}$, as well as the utilization of national health insurance scaling ${ }^{21)}$. Given that scaling is performed by dental hygienists, the wearing of name tags made it possible for the general public to recognize the dental hygienist as a practitioner of scaling, which would have contributed to the increased awareness of the job and the identification of the hygienists themselves ${ }^{18)}$.

The number of inquiries collected by the search keyword "dental hygienist medical assistance" was $21 \%$ of the total number of inquiries collected using all search keywords. Nevertheless, many words directly related to dental treatment procedures were extracted and included in the top 100 most frequently used words, including "chi-lyo," "chungchi," "singyeong," "implant," "gyojeong," "itmom-chi-lyo," "resin," "balchi," "machwi," "crown," and "amalgam". In particular, "chi-lyo," which means treatment, was highly associated with several words, including "singyeong," "resin," and "chungchi". Moreover, the reliability of these associations, i.e., the probability of occurrence of "chi-lyo" in a sentence where the preceding words exist, were relatively high among the 66 association rules (confidence $>0.5$; Table 3), suggesting that these words are often used together in sentences. Therefore, we consider that, in the inquiries about the job of dental hygienists, there is a relatively large demand for information related to dental treatment procedures that the public have previously received or are going to receive in the near future. This supports the previous findings that the most frequently received dental services from dental hygienists are preventive care or medical assistance ${ }^{5,6)}$. However, in terms of the frequency and type of the derived words in the present study, it is worth noting that the terminology related to preventive dental care reflected by scaling is higher in the ranking, but the terminology related to medical assistance work is more quantitative. In addition, fluoride application and sealant have frequently appeared as the jobs of dental hygienists in many studies ${ }^{1)}$, but the words were not detected in the top 100 frequently used words in this study. This result suggests that even 
though preventive dental care is the main job of dental hygienists in the clinical setting, the jobs are not being provided proactively with job autonomy by dental hygienists.

There are some limitations to consider when interpreting the results of this study. First is the consideration for main age group that may generate the collected text data. A previous study investigated inquiries retrieved from Naver "Jishik-iN" using Korean words for dental hygienists as search words, and reported that the proportion of inquiries focused on employment and entrance exams were high, indicating that the collected data were primarily originated from the younger age group $^{11)}$. In the present study, words related to admission to the Department of Dental Hygiene (chiwisaeng-gwa, 16th; chiwisaenghag-gwa, 86th), College (daehag, 24th), and University (daehaggyo, 58th) were extracted as frequently used words, and an association rule was found between the words "chiwisaeng-gwa" and "daehag". Moreover, career-related words such as "chwieob" (employment, 81st) and "jol-eob" (graduation, 88th) were listed in the top 100 frequently used words. Therefore, it is necessary to consider the possibility that the proportion of data formed by young people in their 10s, 20s, and 30s may have been large.

Second, the collection of text data in this study was only performed through one portal Internet site. We will consider the preferences of portal sites and social media channels according to age in future studies in an effort to ensure more comprehensive data.

In summary, the public demand for information related to the job of dental hygienists was mainly related to "chi-lyo" (treatment), "chigwa" (dental clinic), and "ske-illing" (scaling), demonstrating that scaling is recognized as the job of a dental hygienist by the public. However, from the perspective of the job areas of dental hygienists, the higher demand for information related to dental treatment procedures and medical assistance indicates that the public's recognition of overall dental hygienists' job is more focused on medical assistance than preventive dental care that is proactively provided by dental hygienists with job autonomy. The results of this study suggest that the legal scope of the jobs and work of dental hygienists need to be reestablished to provide institutionally safe dental services; indeed, our findings can be used as the basis for granting its justification. In addition, as this study deals with information relating to the job scope of the dental hygienist as recognized by the public, it is expected that the results could be used as an indication to develop strategies for raising public awareness of the occupation of dental hygienists.

\section{Notes}

\section{Conflict of interest}

No potential conflict of interest relevant to this article was reported.

\section{Ethical approval}

This study is a review-based study and does not require an IRB review.

\section{Author contributions}

Conceptualization: Ji-Hyoung Han, Bo-Ra Kim, Eunsuk Ahn, Sun-Mi Kim, Soon-Jeong Jeong, and Soo-Jeong Hwang. Data acquisition: Ji-Hyoung Han, Bo-Ra Kim, Eunsuk Ahn, Sun-Mi Kim, Soon-Jeong Jeong, and Soo-Jeong Hwang. Formal analysis: Bo-Ra Kim, Ji-Hyoung Han, Eunsuk Ahn, Soo-Jeong Hwang, and Sun-Mi Kim. Supervision: Eunsuk Ahn and Ji-Hyoung Han. Writing-original draft: Bo-Ra Kim, Ji-Hyoung Han, Eunsuk Ahn, and Sun-Mi Kim. Writingreview \& editing: Bo-Ra Kim, Eunsuk Ahn, and Sun-Mi Kim.

\section{ORCID}

Bo-Ra Kim, https://orcid.org/0000-0002-3775-280X

Eunsuk Ahn, https://orcid.org/0000-0002-9404-4826

Soo-Jeong Hwang, https://orcid.org/0000-0003-4725-1512

Soon-Jeong Jeong, https://orcid.org/0000-0002-8959-4663

Sun-Mi Kim, https://orcid.org/0000-0003-0718-9419

Ji-Hyoung Han, https://orcid.org/0000-0003-1613-2879

\section{Acknowledgements}

This study was supported by research fund from, Korean Dental Hygienists Association, 2020. 


\section{References}

1. Kim SM, Ahn E, Hwang SJ, Jeong SJ, Kim BR, Han JH: Korean dental hygienists' job description based on systematic review. J Dent Hyg Sci 20: 187-199, 2020. https://doi.org/10.17135/jdhs.2020.20.4.187

2. Kim DY, Lee SH: Recognition of college-preparatory students for dental hygienist. J Korean Soc Dent Hyg 12: 103-111, 2012. https://doi.org/10.13065/jksdh.2012.12.1.103

3. Choi HJ, Park KH: Factors affecting the professional image of dental hygienists. J Korean Soc Dent Hyg 20: 197-207, 2020. https://doi.org/10.13065/jksdh.20200019

4. Lee MR, Ji MG: Study on awareness and images of dental hygienists in some college students. J Korea Enter Ind Assoc 9: 211-218, 2015. https://doi.org/10.21184/jkeia.2015.06.9.2.211

5. Lee KH: Factors affecting perception of dental hygienists. J Korean Soc Dent Hyg 18: 463-476, 2018. https://doi.org/10.13065/jksdh.20180035

6. Park SS, Choo PK: A study on the awareness of dental patients about dental hygienists. J Dent Hyg Sci 10: 191-197, 2010.

7. Kim CT, Yoon HS: Level of dental hygienist awareness and image preference. Asia-pac J Multimed Serv Converg Art Humanit Sociol 6: 623-634, 2016.

8. National Information Society Agency: Internet usage survey of Korea 2019. National Information Society Agency Publishing, Daegu, 2020.

9. Kim JS, Kwon EJ, Song TM: A study on using social big data for expanding analytical knowledge-domestic big data supply-demand expectation. Knowl Manag Res 15: 169-188, 2014.

10. Park JM, Song TM: Identifying strong and emerging signals related to personal debt using social big data. Korean J Soc Welf 72: 35-61, 2020.

http://doi.org/10.20970/kasw.2020.72.1.002

11. Oh SH, Shin NR, Hwang SJ: Analysis of online questions about dental hygienist: case report in Naver intelligent network. Oral Biol Res 42: 62-66, 2018. https://doi.org/10.21851/obr.42.01.201803.62
12. Hwang SJ, Lee SM, Moon HJ, et al.: Searching information on online questions by Korean dental hygienists: case report. J Korean Acad Dent Admin 6: 43-47, 2018.

13. R Core Team: R: A language and environment for statistical computing. R Foundation for Statistical Computing, Vienna, 2020.

14. Yoo H, Min I: An analysis of research trends in social welfare studies in Korea: association rules approach. Health Soc Welf Rev 38: 555-588, 2018. https://doi.org/10.15709/hswr.2018.38.2.555

15. Han YK, Kim SH, Yang JY, Yu JS, Bae SM: A survey of dentists' opinions on the performance of dental hygienists. J Korean Acad Dent Admin 7: 1-9, 2019.

16. Kim YS, Shin MW: A study on the current state and weight of dental hygienists' works. J Korean Soc Dent Hyg 8: 161-175, 2008.

17. Hyeong JH, Jang YJ: The opinions of health care workers on the inclusion of dental hygienists in the category of medical personnel. J Korean Soc Dent Hyg 17: 1013-1024, 2017. https://doi.org/10.13065/jksdh.2017.17.06.1013

18. Kim KJ, Lee G, Jin BH: A survey of the understanding of the scope of work undertaken by dental hygienists and chair-side dental assistants among dental students in Seoul. J Korean Acad Oral Health 43: 224-231, 2019. https://doi.org/10.11149/jkaoh.2019.43.4.224

19. Choi JM, Lee EJ: The effect of the dental patients' perception about dental hygienist on the social demand of dental hygienist. J Dent Hyg Sci 12: 392-403, 2012.

20. Korea Legislation Research Institute: Section 1. Qualification and licenses. Article 42. Names of medical institutions. Retrieved February 2, 2021, from https://elaw.klri.re.kr/kor_ service/lawView.do?hseq=53532\&lang=ENG(2010, January 18).

21. Huh J, Nam S, Lee B, et al.: Improvement of accessibility to dental care due to expansion of national health insurance coverage for scaling in South Korea. J Korean Dent Assoc 57: 644-653, 2019.

22. Jang YE, Kim CB, Kim NH: Utilization of preventive dental services before and after health insurance covered dental scaling in Korea: 2009 to 2014 Community Health Survey. Asia Pac J Public Health 29: 70-80, 2017. https://doi.org/10.1177/1010539516684944 